\title{
A Novel Radiation Enhanced Split-Ring Triplate Antenna
}

\author{
Anne D. Abeygunasekera, Charles E. Free \\ Advanced Technology Institute \\ University of Surrey \\ Guildford, United Kingdom
}

\begin{abstract}
A novel circular polarized antenna structure capable of high radiation efficiency and suitable for use in multilayer microwave circuits is presented. The antenna uses a low permittivity dielectric layer on top of a high permittivity substrate to enhance radiation. The novel split ring design ensures compensation for the progressive loss in the travelling wave around the ring. Data are provided for a prototype antenna working at $20 \mathrm{GHz}$.
\end{abstract}

Keywords-Microwave, Circular Polarization, Travelling wave, Multilayer

\section{INTRODUCTION}

The expanding applications for microwave communication systems have generated a need for compact, high-performance microwave circuits with integrated antennas. In this paper, we propose a novel antenna structure capable of high radiation efficiency suitable for use in multilayer microwave circuits. The traveling wave feed system offers high quality circular polarization which is a key requirement for many existing and developing mobile communication systems. The proposed design can be fabricated using thick film technology, thus satisfying the demand for high volume microwave circuits at low cost.

The proposed antenna uses a low permittivity dielectric layer printed on a high permittivity substrate, as shown in fig. 1 , to improve the fringing fields at the edges of the radiation patches. The work is an extension of the concept using strips of low dielectric constant material underneath the radiation edges of a patch to enhance radiation efficiency [1]. In the proposed design the low dielectric constant material is placed on top of the high permittivity substrate, thus forming a multilayer structure. The antenna comprises three substrate layers with a patch array fed by two half circular microstrip channels, compensating for the progressive loss in signal. A theoretical investigation based on electromagnetic simulation was performed to validate the new radiation efficiency enhanced antenna at $20 \mathrm{GHz}$.

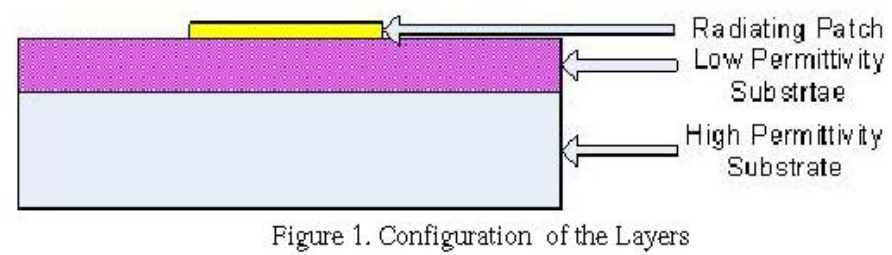

\section{ANTENNA THEORY AND STRUCTURE}

The structure of the radiation enhanced CP antenna is illustrated in fig. 2. The antenna is composed of 8 planar rectangular radiating patches. Each patch is linearly polarized and has dimensions $\mathrm{L}_{\mathrm{p}}$ and $\mathrm{W}_{\mathrm{p}}$, where $\mathrm{L}_{\mathrm{p}}$ is the resonant

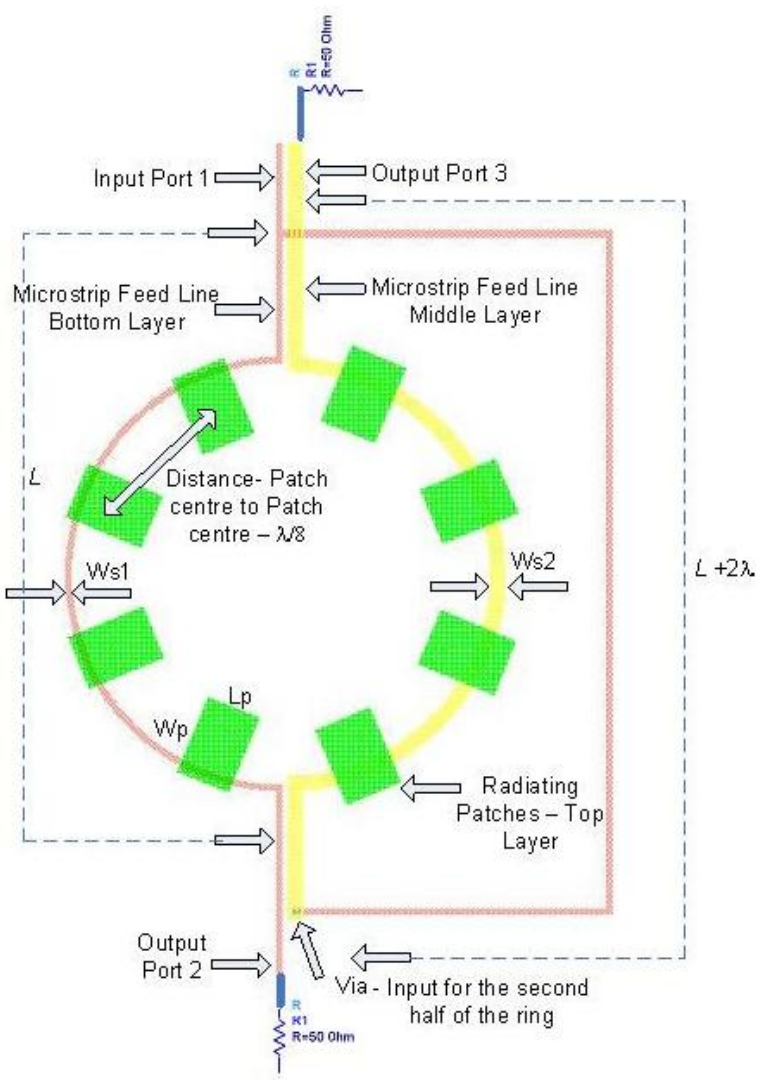

Figure 2. Configuration of the Antenna 
dimension and $\mathrm{W}_{\mathrm{p}}$ is the width of the patch, defining the radiating edges. The patches are excited with a progressive phase lag of $45^{\circ}$ because there is an annular spacing of $\lambda / 8$ between the patches, thus leading to circular polarization. The basic design concept of the antenna was originally established by K. Lum and C. Free[2].

The antenna feed is through a circular $50 \Omega$ microstrip channel, of width $W_{s 1}$ and $W_{s 2}$, which runs on top of the first substrate and on top of the second substrate respectively. The feed is divided into two half rings splitting the power from the feed to the patches. The power in from the feed on the bottom layer is fed through to the feed in the middle layer through a via. Each patch was matched to the respective microstrip line. A portion of the signal travelling in the bottom layer feed line will successively excite each patch. The second half of the patch array is then successively excited through the middle layer feed line, therefore compensating for the progressive loss of signal in the feed.

Simulated data was obtained for a test antenna made on Alumina ADS-96R with the following parameters: substrate thickness $\mathrm{h}=0.254 \mathrm{~mm}$, substrate dielectric constant $\varepsilon_{\mathrm{r}}=9.8$. The key antenna dimensions were:
Length of the patch
Width of the patch

$$
\begin{aligned}
W_{p} & =6.9 \mathrm{~mm} \\
L_{p} & =2.9 \mathrm{~mm}
\end{aligned}
$$

Width of the microstrip line $\mathrm{W}_{\mathrm{s} 1}=0.248 \mathrm{~mm} \quad \mathrm{~W}_{\mathrm{s} 2}=0.578 \mathrm{~mm}$

Diameter of the circular microstrip line channel $=16.4 \mathrm{~mm}$

Circuit dimensions $\quad=50.8 \mathrm{~mm} \times 50.8 \mathrm{~mm}$

As illustrated in fig. 3 a low dielectric constant material was placed beneath the patches to improve the efficiency of the radiation. One of the key parameters affecting radiation efficiency is the dielectric constant. The lower the dielectric constant, the less the concentration of energy in the substrate. Therefore more radiation occurs. The high dielectric constant substrate material reduces the radiation. This is because the most of the EM field is then concentrated in the dielectric between the conductive patch and the ground plane. The low dielectric constant material used was KQ150 (Heraeus Inc) with a dielectric constant of 3.9. Dielectric layer thickness was fabricated to be $50 \mu \mathrm{m}$. The presence of the low dielectric material increases the intensity of the electric field distribution at the radiating edges of the patch.

The coupling between the two microstrip feed lines was minimized by a dielectric layer of $100 \mathrm{um}$. The same low dielectric constant KQ150 (Heraeus Inc) was used to avoid coupling between the microstrip feed lines whilst contributing to the enhancement of radiation.

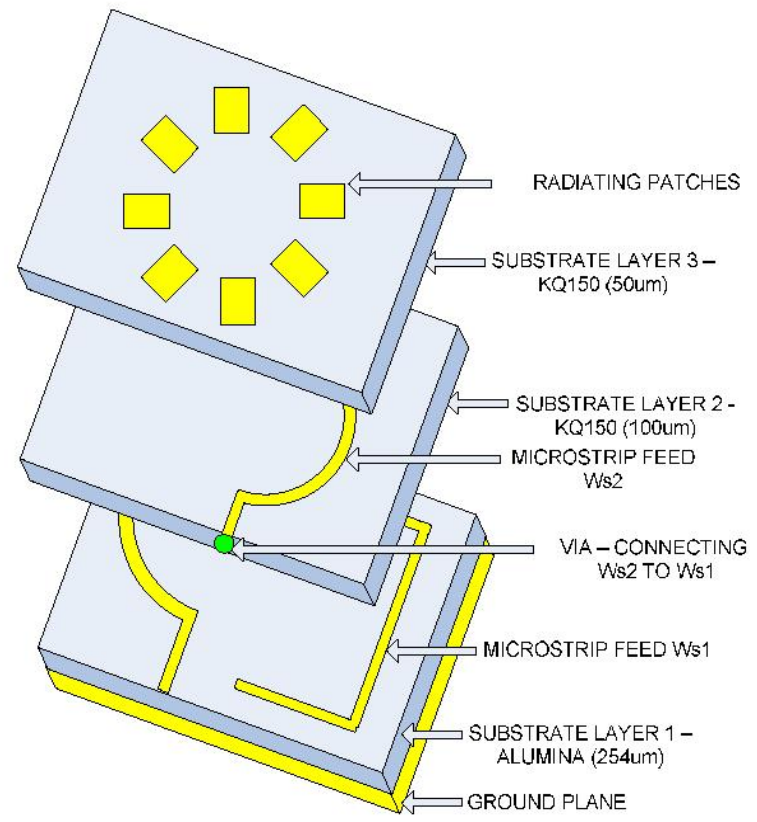

Figure 3. Configuration of the layers

\section{RESULTS - ANTENNA ON CERAMIC}

The structure was modeled using an electromagnetic simulator Momentum $^{\circledR}$ (AGILENT Advanced Design System).

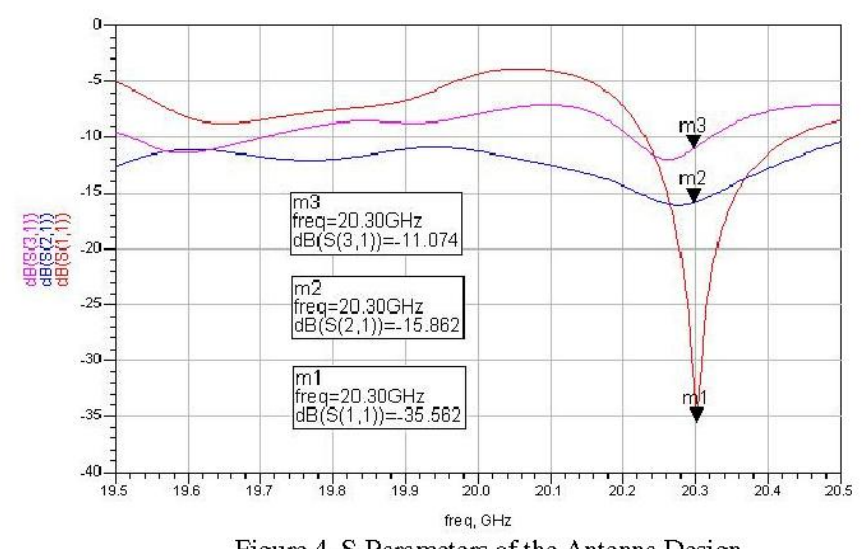

Figure 4. S-Parameters of the Antenna Design

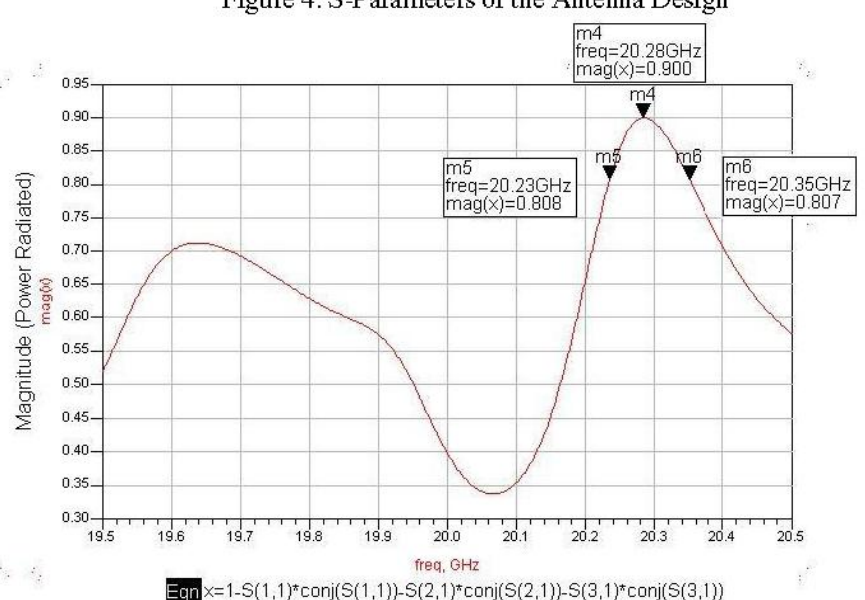

Figure 5. Maximum Power Radiation of the Antenna at the Frequency of Operation 
The optimum frequency of operation was $20.3 \mathrm{GHz}$. This is slightly different from the designed value of $20 \mathrm{GHz}$ because no matching was included to account for the reactance presented by the patches to the feed line. As seen from fig. 4, the return loss of the antenna is $-35.58 \mathrm{~dB}$ at $20.3 \mathrm{GHz}$. In this antenna structure the traveling wave can reflect, radiate or dissipate power in the substrate. By determining S21 and S31, we can find the amount of power dissipated in the antenna. If we assume that the amount of signal dissipated in the substrate is negligible, then S21 and S31 indicate the percentage of power that is radiated. The reflection is negligible, as shown from the high return loss in fig 4 . Therefore most of the signal traveling through the feed has been radiated. S21 and S31 were found to be $-15.86 \mathrm{~dB}$ and $-11.07 \mathrm{~dB}$, respectively, for the above design. Thus, indicating radiation loss between the input port and the respective output ports.

Furthermore, from fig 5. the radiated power from the antenna design was calculated to be $90 \%$ of the input power at the optimum frequency of operation, $20.3 \mathrm{GHz}$. The bandwidth of the antenna was $120 \mathrm{MHz}$. Therefore the design is considered to be useful for practical narrowband applications.

\section{ANTENNA ON POLYMER}

This type of antenna structure was also investigated using polymer material for all three layers. Simulated data was obtained for a test antenna with the following parameters: substrate thickness for each layer $\mathrm{h}=0.127 \mathrm{~mm}$ and substrate dielectric constant $\varepsilon_{\mathrm{r}}=2.5$

The key antenna dimensions were:

Length of the patch $\quad W_{p}=4.6 \mathrm{~mm}$

Width of the patch $\quad L_{p}=4.1 \mathrm{~mm}$

Width of the microstrip line $\mathrm{W}_{\mathrm{s} 1}=0.35 \mathrm{~mm} \quad \mathrm{~W}_{\mathrm{s} 2}=0.744 \mathrm{~mm}$

Diameter of the circular microstrip line channel $=29.6 \mathrm{~mm}$

Circuit dimensions $\quad=35 \mathrm{~mm} \times 50 \mathrm{~mm}$

\section{RESULTS - ANTENNA ON POLYMER}

The structure was modeled using an electromagnetic simulator Momentum $^{\circledR}$ (AGILENT Advanced Design System).

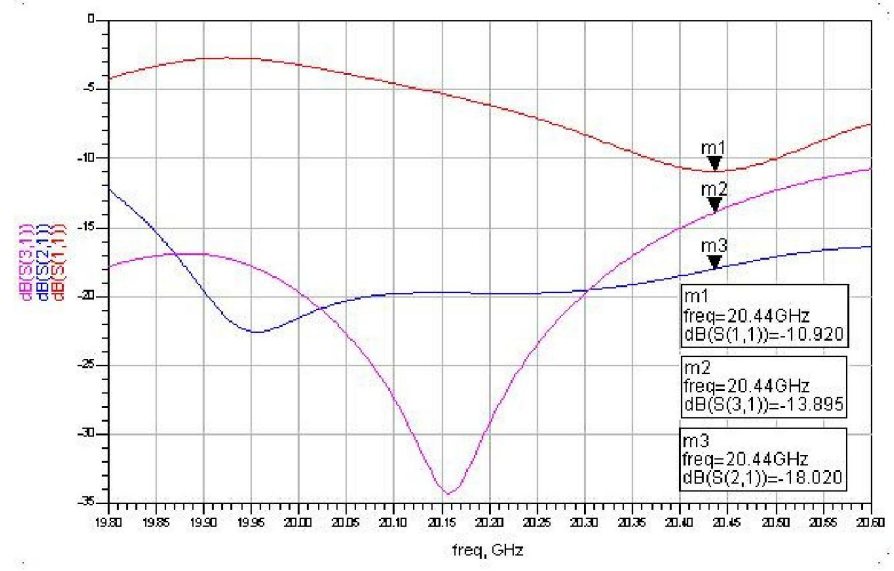

Figure 6. S-Parameters of the Antenna Design

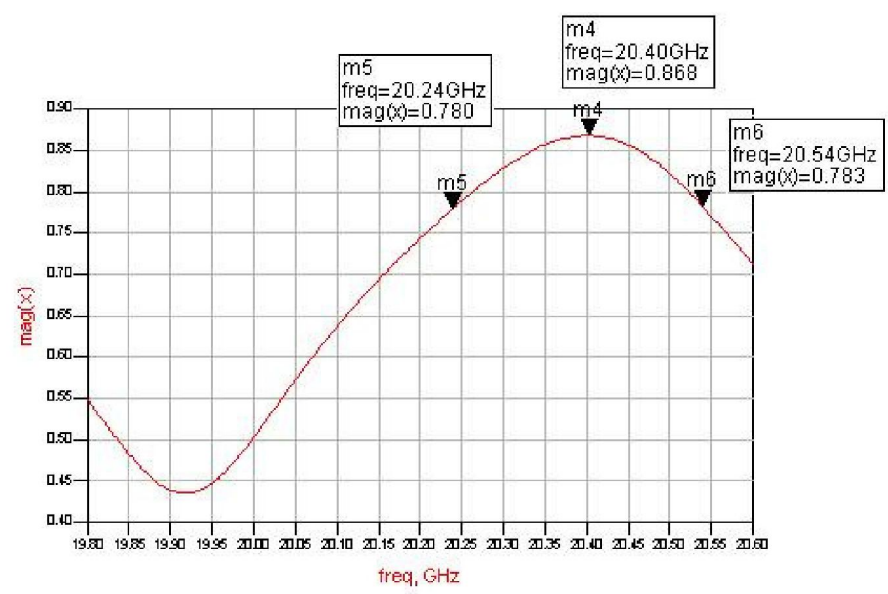

Figure 7. Maximum Power Radiation of the Antenna at the Frequency of Operation

The optimum frequency of operation was $20.44 \mathrm{GHz}$. This is slightly different from the designed value of $20 \mathrm{GHz}$ because no matching was included to account for the reactance presented by the patches to the feed line. As seen from Fig. 6, the return loss of the antenna is $-10.92 \mathrm{~dB}$ at $20.44 \mathrm{GHz}$. The reflection is negligible, as shown from the high return loss in fig 6 . Therefore most of the signal traveling through the feed has been radiated. S21 and S31 are calculated to be $-18.02 \mathrm{~dB}$ and $-13.895 \mathrm{~dB}$ respectively for the above design. Thus, indicating radiation loss between the input port and the respective output ports.

Furthermore, using the data from fig 7 . the radiated power from the antenna design was calculated to be $87 \%$ of the input power at the optimum frequency of operation, $20.4 \mathrm{GHz}$. The bandwidth of the antenna is $300 \mathrm{MHz}$.

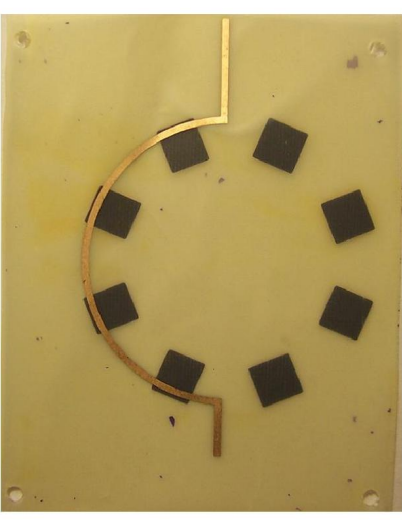

(a) Middle Feed Line

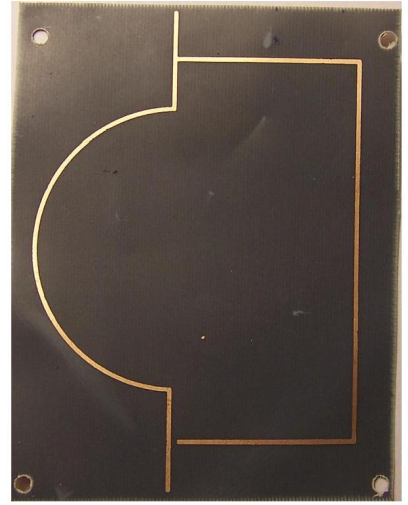

(b) Bottom Feed Line 


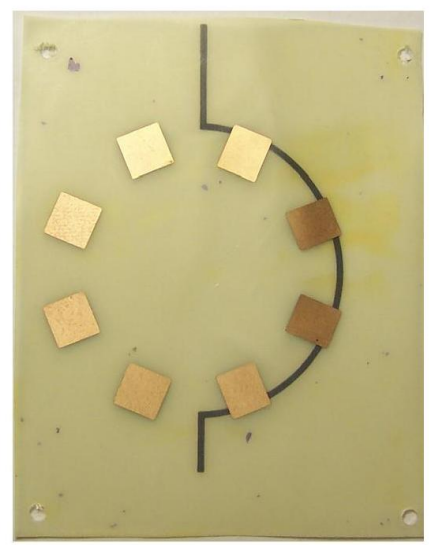

(c) Radiating Patches

Figure 8. Antenna Design

\section{CONCLUSION}

The concept of a novel circular polarized patch antenna has been established. In this paper an extension to the basic antenna design was investigated, and was shown to have beneficial effects. The benefits resulted from radiation enhancement due to the low dielectric constant material located between the patches and the high permittivity supporting substrate. Furthermore, the novel split-ring design ensures compensation for the progressive loss of the signal in traveling around the ring. This form of antenna structure is particularly suitable for inclusion in highly integrated multilayer transceivers.

\section{REFERENCES}

[1] K.M. Lum; T. Tick; C. Free; H. Jantunen, "Design and Measurement Data for a Microwave Dual-CP Antenna Using a New Travelling-Wave Feed Concept", IEEE Trans. On Microwave Theory and Techniques, Vol. 54, no. 6, pp. 2880-2886, June 2006.

[2] K. M. Lum, C. Laohapensaeng, and C. E. Free, "A novel traveling-wave feed technique for circularly polarized planar antennas," IEEE Microwave Wireless Components Letters, vol. 15, no. 3, pp. 180-182, March 2005

[3] K. P. Tang, C. E. Free, and G. F. Goldspink, "A novel slotline-fed microstrin patch antenna", IEEE Antennas and Propagation Conf:, vol. 1, no. 407, pp. 235-238, April 1995.

[4] J. Huang, "A technique for an array to generate circular polarization with linearly polarized elements", IEEE Trans. Antennas and Propagation., vol. 34, no. 9, pp. 1113-1124, September 1986.

[5] E. A. Mariani, C. P. Heinzman, J. P. Agrios, and S. B. Cohn, "Slot line characteristic", IEEE Trans. Microwave Theory Tech., vol. 17, no 12 , pp. 1091-1096, December 1969.

[6] J. B. Knorr, "Slot-line transitions", IEEE Trans. Microwave Theory Tech., vol. 22, no. 5, pp. 548-554, May 1974.

[7] K. M. Lum, and C. E. Free, A Novel Traveling-Wave feed Technique for Circularly Polarized Planar Microstrip antennas, IEEE Antennas and Propagation Conf., vol. 2A, pp. 250 - 253, July 2005. 